\title{
Features of the application of ZigBee technologies in monitoring systems for agricultural objects
}

\author{
Nikolay Boldyrikhin ${ }^{1,}$, Ali Tawafan Al-Obaydi ${ }^{1}$, Alexey Svizhenko ${ }^{2}$, Alexander Manin ${ }^{3}$, \\ and Ivan Sosnovsky ${ }^{3}$ \\ ${ }^{1}$ Don State Technical University, 1, Gagarina sq., Rostov-on-Don, 344003, Russia \\ ${ }^{2}$ Military training centre Southern Federal University, 44, Nekrasovsky lane, Rostov region, 347922, \\ Russia \\ ${ }^{3}$ North Caucasus branch of Moscow Technical University of Communications and Informatics, 62 , \\ Serafimovich st., Rostov-on-Don, 344007, Russia
}

\begin{abstract}
The article discusses the possibilities of using wireless technologies of the Internet of Things at agricultural facilities using the example of ZigBee technologies. This topic seems to be relevant, since the smart farm market is growing very quickly. This is due to the fact that the use of automated control systems for humidity, temperature, acidity, electrical conductivity of the soil, etc. allows you to increase productivity, reduce costs. The aim of the research is to assess the potential communication range between ZigBee devices, and to analyze the factors affecting the quality of the radio channel. As the results of the work, it should be noted a mathematical study of radio channels at frequencies of 868 and $2400 \mathrm{MHz}$, analysis of factors affecting signal propagation. The article also provides practical recommendations for choosing a network topology and ZigBee equipment..
\end{abstract}

\section{Introduction}

The Internet of Things (IoT) is evolving at a rapid pace thanks to the availability of inexpensive hardware. The IoT is affecting our lives today as the volume and variety of big data streams from the IoT expands and analytic technologies develop. Many industries are using the Internet of Things to analyze customer needs in real time, improve agility, improve service, streamline operations, and find innovative ways to work as part of their digital transformation efforts. Technologies of the Internet of Things penetrate into all spheres of our life: science, medicine, education, industry, agriculture [1-11].

According to the portal json.tv, the amount of capital invested in smart farms using IoT technologies was 3 billion euros in 2016 and 4.5 billion by 2020 . The use of smart technologies can significantly increase the productivity of these farms.

Wireless IoT technologies are potentially interesting for implementation in farms [1$8,10,11]$. This is due to the fact that in such farms the facilities are located at a considerable

\footnotetext{
*Corresponding author: boldyrikhin@mail.ru
} 
distance from each other (the area of farms reaches 100 and more hectares). The use of modern wireless technologies is in many ways not inferior in quality to wired ones, but it significantly benefits in cost. With the help of wireless sensors, you can receive information about the state of the soil, moisture, pressure, illumination, temperature, etc. Based on this information, the farmer or an automated system decides on the need for watering, weeding, fertilizing the soil, etc.

At the same time, the use of wireless technologies of the "Internet of Things" has a number of peculiarities due to the terrain, weather conditions, equipment used, etc. The study of these features seems relevant.

\section{Calculation of the parameters of the transmit-receive path of the ZigBee equipment}

At present, three models of radio signal propagation are used to calculate the parameters of the receiving-transmitting path: LOS-model, which takes into account wave propagation only in line of sight (ultrashort waves); propagation model, taking into account the propagation of radio waves due to re-reflection from the earth's surface (long and medium waves); a model that takes into account propagation due to reflection from the upper layers of the atmosphere and from the earth's surface (short waves) [12].

For a preliminary calculation of the distance between the receiver and the transmitter, it is advisable to use the straight-line signal propagation model. Within the framework of this model, the maximum communication range between a receiver and a transmitter located near the Earth's surface depends on the curvature of the planet (the radius of the planet is $6365 \mathrm{~km}$ ), as well as on the height of the transmitting and receiving antennas. This distance in kilometers is calculated using the following formula:

$$
L_{\max }=\frac{\left(\sqrt{2 h_{1} R z}+\sqrt{2 h_{2} R z}\right)}{1000},
$$

where $h_{1}$ is the height of the transmitting antenna, $\mathrm{m} ; h_{2}$ - receiving antenna height, $\mathrm{m}$; $R z$ - radius of the Earth, $\mathrm{m}$.

Channel budget. The budget of a radio channel is approximately determined by the difference between the power of the transmitting device and the sensitivity limit of the receiving device:

$$
B \approx P_{t r}-\vartheta_{\text {rec }},
$$

where $P_{t r}$ is the transmitter power, $\mathrm{dBm} ; \vartheta_{r e c}$ - receiver sensitivity, - $\mathrm{dBm}$.

With this rough estimate, with a $+10 \mathrm{dBm}$ transmitter and a $110 \mathrm{dBm}$ receiver, we get an approximate $120 \mathrm{~dB}$ channel budget. Of course, for a more realistic budget estimate, the antenna gain must be taken into account:

$$
B \approx P_{t r}+G_{t r}-\vartheta_{r e c}+G_{r e c},
$$

where $P_{t r}$ is the transmitter power, $\mathrm{dBm} ; \vartheta_{\text {rec }}$ - receiver sensitivity, - $\mathrm{dBm} ; G_{t r}$ transmitting antenna gain, $\mathrm{dBi} ; G_{r e c}$ is the gain of the receiving antenna, dBi.

The receive antenna gain is generally assumed to be $2.1 \mathrm{dBi}$. Note that the gain can also be expressed in decibels, with the $\mathrm{dB}$ and $\mathrm{dBi}$ values being the same. The channel budget for such antennas under the conditions of the previous example will be $124.2 \mathrm{~dB}$. 


\begin{tabular}{|c|c|c|}
\hline $\begin{array}{l}\text { The operating frequency of the } \\
\text { transmitter }\end{array}$ & The distance between the points & $\begin{array}{l}\text { The losses in the cable and the } \\
\text { connectors }\end{array}$ \\
\hline$(\mathrm{MHz})$ & $(\mathrm{km})$ & $(\mathrm{dB})$ \\
\hline Transmitter power & Transmitter antenna gain & Loss of penetration \\
\hline $16 \quad(\mathrm{dBm})$ & $\nabla(\mathrm{dBi})$ & $\square(\mathrm{dB})$ \\
\hline The receiver sensitivity & Receiver antenna gain & Fading margin \\
\hline$(-\mathrm{dBm})$ & $(\mathrm{dBi})$ & (dB) \\
\hline The free space loss & $\begin{array}{l}\text { Signal strength at the receiver } \\
\text { input }\end{array}$ & Forecast operation of the radio \\
\hline 125.1 (dB) & $-106.1 \quad(\mathrm{dBm})$ & An unstable connection \\
\hline $\begin{array}{l}\text { The operating frequency of the } \\
\text { transmitter }\end{array}$ & The distance between the points & $\begin{array}{l}\text { The losses in the cable and the } \\
\text { connectors }\end{array}$ \\
\hline$(\mathrm{MHz})$ & $(\mathrm{km})$ & $\square(\mathrm{dB})$ \\
\hline Transmitter power & Transmitter antenna gain & Loss of penetration \\
\hline$(\mathrm{dBm})$ & $(\mathrm{dBi})$ & $(\mathrm{dB})$ \\
\hline The receiver sensitivity & Receiver antenna gain & Fading margin \\
\hline$(-\mathrm{dBm})$ & $(\mathrm{dBi})$ & $(\mathrm{dB})$ \\
\hline The free space loss & $\begin{array}{l}\text { Signal strength at the receiver } \\
\text { input }\end{array}$ & Forecast operation of the radio \\
\hline $122.5 \quad(\mathrm{~dB})$ & $\square(\mathrm{dBm})$ & Medium quality \\
\hline $\begin{array}{l}\text { The operating frequency of the } \\
\text { transmitter }\end{array}$ & The distance between the points & $\begin{array}{l}\text { The losses in the cable and the } \\
\text { connectors }\end{array}$ \\
\hline $868 \quad(\mathrm{MHz})$ & $\square(\mathrm{km})$ & $1 \quad(\mathrm{~dB})$ \\
\hline Transmitter power & Transmitter antenna gain & Loss of penetration \\
\hline$(\mathrm{dBm})$ & $(\mathrm{dBi})$ & $0 \quad(\mathrm{~dB})$ \\
\hline The receiver sensitivity & Receiver antenna gain & Fading margin \\
\hline$(-\mathrm{dBm})$ & (dBi) & $\square(\mathrm{dB})$ \\
\hline The free space loss & $\begin{array}{c}\text { Signal strength at the receiver } \\
\text { input }\end{array}$ & Forecast operation of the radio \\
\hline 118.8 (dB) & $\square(\mathrm{dBm})$ & Good quality \\
\hline $\begin{array}{l}\text { The operating frequency of the } \\
\text { transmitter }\end{array}$ & The distance between the points & $\begin{array}{l}\text { The losses in the cable and the } \\
\text { connectors }\end{array}$ \\
\hline$(\mathrm{MHz})$ & $(\mathrm{km})$ & 1 \\
\hline Transmitter power & Transmitter antenna gain & Loss of penetration \\
\hline$(\mathrm{dBm})$ & $(\mathrm{dBi})$ & $(\mathrm{dB})$ \\
\hline The receiver sensitivity & Receiver antenna gain & Fading margin \\
\hline$-110 \quad(-d B m)$ & $(\mathrm{dBi})$ & 20.9 (dB) \\
\hline The free space loss & $\begin{array}{l}\text { Signal strength at the receiver } \\
\text { input }\end{array}$ & Forecast operation of the radio \\
\hline $108.1 \quad(d B)$ & $-89.1 \quad(\mathrm{dBm})$ & Excellent signal \\
\hline
\end{tabular}

Fig. 1. Parameters of the radio channel for various initial data.

The permitted power of modern ZigBee transmitters ranges from 1-100 $\mathrm{mW}(0.01-20$ $\mathrm{dBm}$ ), and the sensitivity ranges from $-90 \mathrm{~mW}$ to $-110 \mathrm{~mW}$. In accordance with (4), the channel budgets for these values will be: 


$$
\begin{aligned}
& B_{\min } \approx 0.01+2.1+90+2.1=94.21 \mathrm{~dB} \\
& B_{\max } \approx 20+2.1+110+2.1=134.2 \mathrm{~dB} .
\end{aligned}
$$

There are more accurate methods for calculating the budget. For example, an online calculator is provided at http://bwa.lgp.kz that allows you to take into account cable losses, penetration losses, etc. (Figure 1).

Figure 1 shows the results of estimating the parameters of the radio channel for various distances between the receiver and the ZigBee transmitter operating at the $868 \mathrm{MHz}$ carrier frequency. As you can see from the figure: transmitter power and receiver sensitivity with specified maximum values (for a given frequency). The $868 \mathrm{MHz}$ frequency is one of the most popular when deploying a radio channel outside populated areas, since it allows you to organize communication over sufficiently long distances and use a less noisy part of the range. Theoretically, if you use a lower carrier frequency, the communication range will increase, however, as the frequency decreases, the level of interference increases, which negatively affects the quality of the communication channel. The data transfer rate is also significantly reduced. If you use a $2.4 \mathrm{GHz}$ carrier, the communication range is significantly reduced, power consumption increases, but the data transfer rate increases significantly. Figure 2 shows the parameters of the radio channel for the $2.4 \mathrm{GHz}$ frequency.

Analyzing Figures 1 and 2, it can be seen that for a radio channel at a frequency of 868 $\mathrm{MHz}$, the average quality is provided at a distance of $37 \mathrm{~km}$, and for a $2.4 \mathrm{GHz}$ radio channel, this distance is $15 \mathrm{~km}$, excellent signal quality is provided at distances of $7 \mathrm{~km}$ and $2 \mathrm{~km}$, respectively.

A radio channel operating at a frequency of $868 \mathrm{MHz}$ can provide a stable connection for a long time, not only in line-of-sight conditions, but also in urban areas.

The increase in the communication range compared to the $2.4 \mathrm{GHz}$ band is associated with the possibility of using a narrower receiver bandwidth, which allows a significant increase in sensitivity compared to $2.4 \mathrm{GHz}$ chips, in which the bandwidth cannot be narrowed due to the greater instability of the quartz resonator at high frequencies. However, narrowing the bandwidth results in lower data rates.

It is also important to note that when radio waves pass various obstacles, their energy is significantly reduced, while lower-frequency waves are attenuated much less, which is very important in indoor conditions or in the presence of natural obstacles.

To ensure good quality of the radio channel, it is necessary that the budget of the radio link is at least $10 \ldots 20 \mathrm{~dB}$. The spread of this parameter is due to additional factors affecting the quality of communication: the type of modulation, the use of redundant coding, etc.

An important property of sub-gigahertz waves is that they are more diffracted than waves in the $2.4 \mathrm{GHz}$ range; at $868 \mathrm{MHz}$, they better avoid obstacles. To ensure highquality communication, it is necessary that the signal energy at the receiver input exceed the noise value, which is always present in space and is mainly due to man-made factors: radiation from a huge number of transmitters for various purposes, as well as radiation from industrial installations, etc. The natural natural radio noise generated by astronomical objects such as the Sun, supernova remnants, pulsars, quasars and other objects cannot be discounted either. Therefore, the signal level must significantly exceed the noise level. Sometimes this requirement is specified in the specifications as the required signal-to-noise ratio $(\mathrm{S} / \mathrm{N})$. It is important to take into account, in addition to the transmitter power and the receiver sensitivity, the attenuation of the radio signal in the atmosphere. 


\begin{tabular}{|c|c|c|}
\hline $\begin{array}{l}\text { The operating frequency of the } \\
\text { transmitter }\end{array}$ & The distance between the points & $\begin{array}{l}\text { The losses in the cable and the } \\
\text { connectors }\end{array}$ \\
\hline$\square(\mathrm{MHz})$ & $\square(\mathrm{km})$ & $(\mathrm{dB})$ \\
\hline Transmitter power & Transmitter antenna gain & Loss of penetration \\
\hline $25 \quad(\mathrm{dBm})$ & $(\mathrm{dBi})$ & $(\mathrm{dB})$ \\
\hline The receiver sensitivity & Receiver antenna gain & Fading margin \\
\hline$(-\mathrm{dBm})$ & $D(\mathrm{dBi})$ & $\square(\mathrm{dB})$ \\
\hline The free space loss & $\begin{array}{c}\text { Signal strength at the receiver } \\
\text { input }\end{array}$ & Forecast operation of the radio \\
\hline $128(\mathrm{~dB})$ & $\square(\mathrm{dBm})$ & An unstable connection \\
\hline $\begin{array}{l}\text { The operating frequency of the } \\
\text { transmitter }\end{array}$ & The distance between the points & $\begin{array}{l}\text { The losses in the cable and the } \\
\text { connectors }\end{array}$ \\
\hline $2400 \quad(M H z)$ & $\square(\mathrm{km})$ & $\square(\mathrm{dB})$ \\
\hline Transmitter power & Transmitter antenna gain & Loss of penetration \\
\hline$(\mathrm{dBm})$ & $(\mathrm{dBi})$ & $(\mathrm{dB})$ \\
\hline The receiver sensitivity & Receiver antenna gain & Fading margin \\
\hline$-102(-d B m)$ & $(\mathrm{dBi})$ & $6.5 \quad(\mathrm{~dB})$ \\
\hline The free space loss & $\begin{array}{l}\text { Signal strength at the receiver } \\
\text { input }\end{array}$ & Forecast operation of the radio \\
\hline $123.5(\mathrm{~dB})$ & $-95.5 \quad(\mathrm{dBm})$ & Medium quality \\
\hline $\begin{array}{l}\text { The operating frequency of the } \\
\text { transmitter }\end{array}$ & The distance between the points & $\begin{array}{c}\text { The losses in the cable and the } \\
\text { connectors }\end{array}$ \\
\hline $2400 \quad(\mathrm{MHz})$ & $(\mathrm{km})$ & $(\mathrm{dB})$ \\
\hline Transmitter power & Transmitter antenna gain & Loss of penetration \\
\hline $25(\mathrm{dBm})$ & $(\mathrm{dBi})$ & $0 \quad(d B)$ \\
\hline The receiver sensitivity & Receiver antenna gain & Fading margin \\
\hline$-102 \quad(-d B m)$ & (dBi) & $10.9(\mathrm{~dB})$ \\
\hline The free space loss & $\begin{array}{c}\text { Signal strength at the receiver } \\
\text { input }\end{array}$ & Forecast operation of the radio \\
\hline 119.1 (dB) & $\square(\mathrm{dBm})$ & Good quality \\
\hline $\begin{array}{l}\text { The operating frequency of the } \\
\text { transmitter }\end{array}$ & The distance between the points & $\begin{array}{l}\text { The losses in the cable and the } \\
\text { connectors }\end{array}$ \\
\hline $2400 \quad(\mathrm{MHz})$ & $(\mathrm{km})$ & $(\mathrm{dB})$ \\
\hline Transmitter power & Transmitter antenna gain & Loss of penetration \\
\hline$(\mathrm{dBm})$ & (dBi) & $0 \quad(d B)$ \\
\hline The receiver sensitivity & Receiver antenna gain & Fading margin \\
\hline$(-\mathrm{dBm})$ & $(\mathrm{dBi})$ & $\square(\mathrm{dB})$ \\
\hline The free space loss & $\begin{array}{c}\text { Signal strength at the receiver } \\
\text { input }\end{array}$ & Forecast operation of the radio \\
\hline $106 \quad(\mathrm{~dB})$ & $(\mathrm{dBm})$ & Excellent signal \\
\hline
\end{tabular}

Fig. 2. Parameters of the radio channel when operating at a frequency of $2.4 \mathrm{GHz}$.

It should be noted that at lower frequencies the attenuation of the signal in the atmosphere is lower than at higher frequencies, which also has a positive effect on the communication range.

The considered calculator makes it possible to calculate the parameters of the radio channel and determine whether communication is possible under given conditions, as well 
as which antennas should be used. At the same time, this method does not take into account the height of the antennas, the effect of distance on the blocking of Fresnel zones due to the curvature of the Earth's surface and the features of the relief between the points of the radio path.

For a more realistic assessment of the quality of the communication channel and the permissible distance between the receiver and transmitter, it is necessary to additionally take into account a number of factors. When a transmitting antenna radiates a radio wave, it propagates not only in the direction of the receiver, but also in other directions, is reflected from various objects, including from the Earth's surface, as a result, an interference phenomenon occurs at the receiving point, which can lead to a deterioration in the quality of communication. If the receiver or transmitter is located on mobile objects, or both are mobile, then the task of determining realistic parameters becomes much more difficult. Of course, all these factors are problematic to take into account, but the two-beam model makes it possible to take into account the influence of the signal reflected from the Earth's surface. It should be noted that the dual-beam model is currently very popular among designers of wireless communication lines. It formed the basis of many software environments that simulate the radio link. The use of this model makes it possible to calculate the parameters of the radio channel rather realistically [12].

\section{Choosing ZigBee equipment}

The ZigBee standard is a wireless technology designed specifically to transmit small traffic over relatively short distances while minimizing hardware power consumption.

The ZigBee specification provides for the presence of three types of devices in the network: coordinators, routers and terminal devices [9, 12-15] (Figure 3).

Coordinators manage subordinate network nodes, store information about configuration files, define frequency channels, and set network identifiers. They can act as sources, receivers and repeaters of messages.

Routers determine the route of delivery of a message, and some routers can also optimize routes to specific points.

Terminal devices cannot be repeaters, but act only as a source or receiver of messages.

When constructing a receiving-transmitting path, the communication range will be of decisive importance and significantly affect the cost, complexity and speed of project implementation. Therefore, the developer, as a rule, tries to optimize the parameters of the receiver and transmitter in order to achieve the maximum possible distance, which allows reducing the number of relay nodes or avoiding their use altogether. The height of the antennas has a very significant effect on the communication range, but at the same time, it can significantly affect the cost of the project. If the range of radio communication is not a determining parameter, then developers and installers try to reduce the power of the transmitter to save energy of the power supply.

Before proceeding to the choice of equipment, you need to decide on the manufacturer of the ZigBee standard equipment. This is due to the fact that ZigBee receivers and transmitters work in tandem with controllers, they can also be additionally equipped with antenna amplifiers, software, so it is advisable to use equipment from one manufacturer that is well compatible with each other [9, 12-15].

Currently, a fairly large number of ZigBee equipment manufacturers are represented on the Russian market: NXP, Ember, Freescale, Telecontrolli, Analog Devices, Atmel, Pt, TI, Maxstream. Their products are made using roughly similar technology and differ in memory size, feature set and price. TI products are among the most advanced in terms of technical parameters and ready-made software. In wireless solutions, TI competes with different manufacturers, depending on the specific application [12, 14,15]. TI's ZigBee 
components are highly-specced and are therefore popular around the world. Proof of this is the fact that independent ZigBee module manufacturers such as Panasonic and Radiacrafts make their own radio modules based on TI chips. It should also be noted that TI, as a supplier of low power microcontrollers, has excellent potential to significantly increase the runtime of battery-powered ZigBee devices.

\section{CLUSTER TREE}

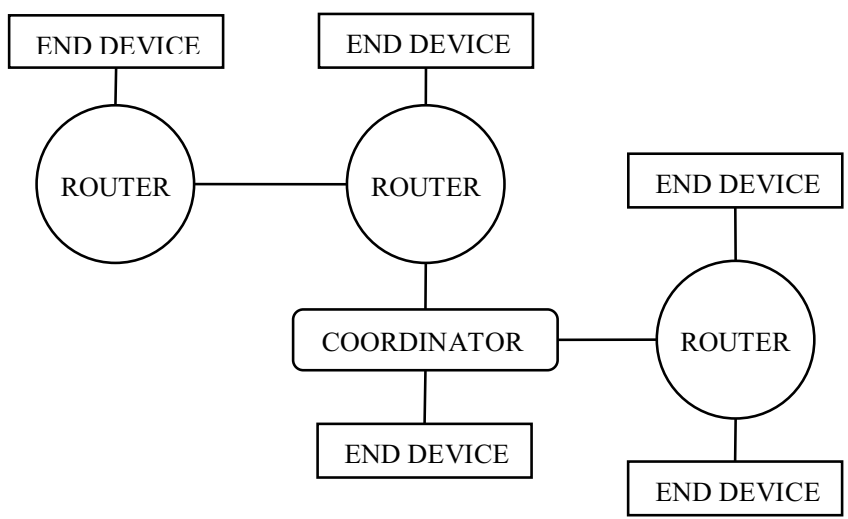

\section{MULTI-CELL NETWORK}

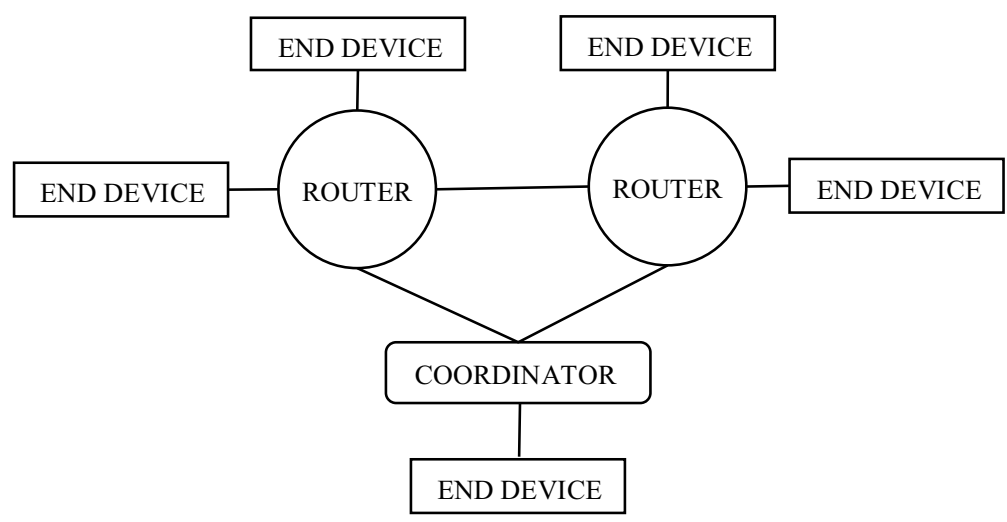

STAR

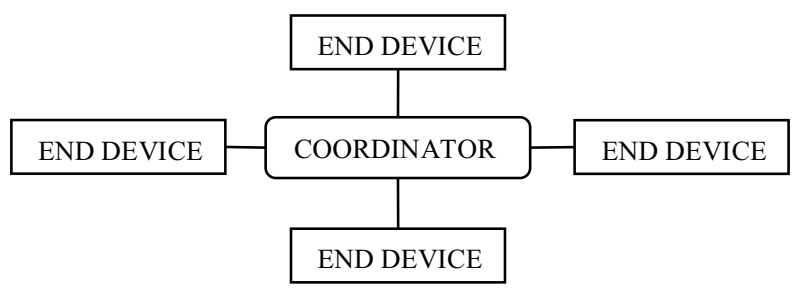

Fig. 3. Topologies of ZigBee networks.

Before making the final choice of equipment, it is necessary to specify the features of the automated control system. It is assumed that the considered automated monitoring and 
control system is significantly distributed over the territory, which is typical for agricultural facilities.

When building an automated control system, the cluster tree topology (Fig. 1) is well suited for objects of complex structure, objects elongated in length. The multi-cell network and star topologies work well for margins as they cover well the areas of simple shapes.

After choosing the topology, you need to decide: at what frequency to create a radio channel: $868 \mathrm{MHz}$ or $2.4 \mathrm{GHz}$. It should be noted that at low data transmission rates and at long distances, it is advisable to use a radio channel at a frequency of $868 \mathrm{MHz}$. Among the devices operating at frequencies below $1 \mathrm{GHz}$, the $\mathrm{CC} 112 \mathrm{x}$ family should be distinguished, which have good sensitivity (from -117 to $-129 \mathrm{dBm}$ ), the output power varies in the range from 14 to $16 \mathrm{dBm}$. A simple calculation of the channel budget gives a value in the order of $140 \mathrm{dBm}$, which provides very good range [12]. In this series, the wake up mode from the sleep state (wake up by the presence of a radio signal) has been improved, which has led to a significant reduction in power consumption. In addition to the energy-saving properties of CC112x microcircuits, a new radio channel listening mode (the so-called Channel Sniff Mode) has been introduced. To enter the listening mode, an interval equal to the transmission time of four bits of the packet preamble is required. During this time, the receiver performs auto-tuning and automatic gain control. In this mode, the quick set capability is combined with a longer preamble to automatically periodically check for signal presence. In this case, the receiver periodically checks for the presence of a signal in the channel, then turns off again. Using the listening mode does not affect the characteristics of the microcircuit, such as sensitivity, selectivity, etc., but allows you to significantly save the power supply. In general, the power consumption of the receiver will be affected by the data transmission rate, the preamble length, but the gain is provided even at high transmission rates. Using the listen mode when using a 4-bit preamble and at a speed of $1.2 \mathrm{kbps}$ allows you to reduce the current consumption from $21 \mathrm{~mA}$ to $3 \mathrm{~mA}$, and at a speed of $50 \mathrm{kbps}$, the current is reduced to $16 \mathrm{~mA}$.

Microcircuits CC112x meet the highest requirements when organizing control systems for geographically dispersed objects.

A significant factor that reduces development costs is the availability of ready-made hardware solutions for network radio modules.

The CC112x family is deservedly one of the most demanded in the development of systems in the range of less than $1 \mathrm{GHz}$, therefore Texas Instruments pays great attention to further development of this direction.

Analyzing the parameters of the $\mathrm{CC} 112 \mathrm{x}$ microcircuits, it can be seen that they are in many ways similar, however, the $\mathrm{CC} 1120$ microcircuit has a higher noise reduction, better sensitivity, and a lower minimum frequency channel bandwidth, therefore its use is more appropriate in many cases, since with the same transmitter power, higher sensitivity and noise reduction will allow you to win in communication range.

\section{Conclusion}

As a result of the studies carried out, it can be concluded that the optimal radio channel for agricultural objects is a radio channel at a frequency of $868 \mathrm{MHz}$. Its use makes it possible to create a high quality radio link with a length of up to $7000 \mathrm{~m}$. Obviously, this length may not be enough. In this case, it is necessary to additionally use repeater routers.

As a practical recommendation for choosing hardware for an automated control system for geographically distributed equipment, CC1120 transceivers are offered, with high quality characteristics for organizing a sub-GHz radio channel. 


\section{References}

1. Yi Li, Yang Zheng, Microprocessors and Microsystems 82, 103852 (2021) https://doi.org/10.1016/j.micpro.2021.103852

2. Jiangchuan Fan, Ying Zhang, Weiliang Wen, et al., Journal of Cleaner Production 280(1), 123651 (2020) https://doi.org/10.1016/j.jclepro.2020.123651

3. S. Kirana, S. Sandeep Kanumallib, K. Venkata Sesha Sai Rama Krishnab, N.Chandrac, Materials Today: Proceedings (2021) https://doi.org/10.1016/j.matpr.2020.11.081

4. A. Sinha, G. Shrivastava, P. Kumar, Sustainable Computing: Informatics and Systems 8(23), 88-102 (2019) https://doi.org/10.1016/j.suscom.2019.07.001

5. Keoma Brun-Lagunaa, Ana Laura Diedrichsbe, Diego Dujovnec et al., Computer Communications 121, 83-90 (2018) https://doi.org/10.1016/j.comcom.2018.03.010

6. Jie Xiao, Jing Tao Li, Procedia Computer Science 166, 419-422 (2020) https://doi.org/10.1016/j.procs.2020.02.072

7. O.P. Bodunde, U.C. Adie, A.A. Aderoba, et al., Computers and Electronics in Agriculture (2019) https://doi.org/10.1016/j.compag.2019.03.021

8. S. Sadowski, P. Spachos, Computers and Electronics in Agriculture 172, 105338 (2020) https://doi.org/10.1016/j.compag.2020.105338

9. S. Choudhury, P. Kuchhal, R. Singh, Procedia Computer Science 48, 367-372 (2015) https://doi.org/10.1016/j.procs.2015.04.195

10. S. Sadowski, P. Spachos, Computers and Electronics in Agriculture 172, 105338 (2020) https://doi.org/10.1016/j.compag.2020.105338

11. Sh. Li, Sh. Peng, W. Chen, X. Lu, Computer Communications 36, 459-467 (2013)

12. R. Wallace, Achieving Optimum Radio Range SWRA479A (2015) https://www.ti.com/lit/an/swra479a/swra479a.pdf?ts=1611924932961

13. T.V. Chien, H.N. Chan, T.N. Huu, International Journal on Advanced Science, Engineering and Information Technology 2(1), 70-74 (2011)

14. L. Krzak, IFAC-Papers OnLine 48(4), 290-295 https://doi.org/10.1016/j.ifacol.2015.07.049

15. A.M. Albertia, D. Mazzerb, M.M. Bontempob, et al., Computers \& Electrical Engineering 57, 147-161 (2016) https://doi.org/10.1016/j.compeleceng.2016.07.008 